CDD: 113

\title{
Sobre la crítica de Mumford al realismo nomológico ${ }^{1}$
}

\author{
Bruno Borge \\ Universidad de Buenos Aires \\ Departamento de Filosofía \\ Ciudad Autónoma de Buenos Aires, Argentina \\ brunojborge@gmail.com
}

Received: 03.12.2014; Revised: 03.11.2015; Accepted: 11.11.2015

DOI: http://dx.doi.org/10.1590/0100-6045.2015.V38N3.BB

Resumen: Stephen Mumford (2004) propone una alternativa al Realismo Nomológico que no requiere resignar los compromisos modales comúnmente asociados a posiciones realistas, un Realismo Sin Leyes (RSL) donde el peso de la modalidad no está puesto sobre las leyes sino sobre propiedades entendidas en términos de poderes y disposiciones. Las ventajas relativas del RSL dependen, sin embargo, de una serie de objeciones que presenta al RN. La principal de ellas es la que Mumford denomina el Dilema Central, según el cual el RN no es capaz de dar cuenta de cómo las leyes gobiernas sus instancias. En el presente trabajo me propongo mostrar que ni el Dilema Central ni el resto de los argumentos de Mumford contra el RN justifican su rechazo.

Palabras clave: Leyes naturales; leyes científicas; Realismo Nomológico.

\section{On Mumford's critic against Nomological Realism}

\begin{abstract}
Stephen Mumford (2004) proposes an alternative to Nomological Realism (NR) compatible with the modal commitments commonly associated to realist positions, a Realist Lawlessness (RL) in which the weight of modality is not set on laws but on properties, understood in terms of powers and propensities. However, RL's

\footnotetext{
${ }^{1}$ El presente trabajo fue realizado con el apoyo de la Agencia Nacional de Promoción Científica y Tecnológica y la Universidad de Buenos Aires. Agradezco a dos evaluadores anónimos de Manuscrito, cuyos comentarios contribuyeron a mejorar la versión final de este artículos.
} 
relative merits depend on a series of objections presented to NR. The main one is the so-called Central Dilemma, according to which NR is unable to give an account of how laws govern their instances. In this paper, I aim to show that neither the Central Dilemma nor the rest of Mumford's arguments against NR justify its rejection.

Keywords: Natural laws; scientific laws; Nomological Realism.

\section{Introducción}

La cuestión de las leyes naturales ha sido un tópico de profundo interés para la ciencia moderna y, por supuesto, para la filosofía que la tuvo por su objeto de indagación. Con todo, su análisis sistemático es relativamente reciente. Incluso en el marco del debate Realismo/Antirrealismo Científicos el estatus de las leyes de la naturaleza no fue discutido, la mayoría de las veces, sino de modo lateral. Los acercamientos más tempranos - e.g. Chisholm (1946; 1955), Goodman (1947), y Hempel y Oppenheim (1948) - han tendido a priorizar un enfoque lógico-sintáctico, centrándose así en la posibilidad de distinguir dentro de la familia de los enunciados legaliformes a las auténticas leyes de las meras generalizaciones accidentales. Sin embargo, el tratamiento del trasfondo metafísico de la distinción fue frecuentemente omitido, o dado por sentado como una simple disputa entre humeanos y necesitaristas. Si bien ese rasgo puede adjudicarse a la influencia más o menos patente del Empirismo Lógico, cuya reticencia hacia las cuestiones metafísicas es bien conocida, incluso en instancias más cercanas a las polémicas actuales - y tal vez en parte como reflejo de lo anterior- las nociones de 'ley científica' (un enunciado universal, irrestricto, etc., postulado por la ciencia) y 'ley natural' (el hacedor de verdad del enunciado legal) son frecuentemente confundidas o tratadas como una sola.

El debate contemporáneo, por el contrario, asume una forma más compleja que admite variados matices. Nadie duda seriamente, a pesar de todo, de que haya regularidades en el mundo. Nadie, seriamente, pone en cuestión que si un enunciado describe adecuadamente una de esas regularidades en el pasado, presente y futuro (un enunciado que será universal, irrestricto y verdadero), merece ser llamado 'ley'. El punto conflictivo es en virtud de qué ese enunciado sería verdadero, es decir, si existe efectivamente un sustrato objetivo que haga de esa regularidad algo necesario, y no meramente una feliz 
coincidencia cósmica. Algunos (creo honestamente que es mucho más complicado de lo que parece saber cuántos) han encontrado en las leyes ese sustrato, ellas no son meras proyecciones de nuestra creatividad sino algo en la naturaleza, son cosas que descubrimos como soporte de la necesidad objetiva de las regularidades, es decir, como su principio tanto explicativo como ontológico. Ellos, sean quiénes y cuántos fueren, son los defensores del Realismo Nomológico (RN). Debe destacarse, sin embargo, que postular conexiones necesarias entre los fenómenos no es condición suficiente para sostener el RN, debe sostenerse que son las leyes naturales y no otra cosa el fundamento ontológico de tal necesidad, es por ello que tanto posiciones humeanas como anti-humeanas pueden convivir bajo el abrigo del Antirrealismo Nomológico (AN).

Stephen Mumford dedica su Laws in Nature (2004) a ilustrar ese punto, a mostrar, en resumidas cuentas, que negar que las leyes existan no implica que debamos contentarnos con una metafísica de simples conjunciones constantes, donde la necesidad es sólo un hábito de nuestras mentes mal tomado por principio. La propuesta de Mumford tiene como propósito hacer patente que las leyes son tan sólo un candidato posible para explicar tanto las regularidades como los vínculos de necesitación que las constituyen. No son el único candidato ni mucho menos el mejor, son de hecho un mal candidato; y las razones que esgrime en favor de ese punto gravitan en torno a lo que da en llamar el Dilema Central: o bien las leyes son internas a sus instancias, o bien son externas a ellas; pero resulta que tanto si las consideramos internas como externas a sus instancias, no es posible dar cuenta de cómo ellas gobiernan aquellos fenómenos que caen bajo su cobertura, por lo que la posibilidad de considerarlas como fundamento de la regularidad quedaría clausurada. Pero hay más: considerarlas externas a sus instancias nos lleva a sostener un quidditismo respecto de la naturaleza de las propiedades, y considerarlas internas requiere de postular la existencia de propiedades esenciales a las clases, lo que deriva en un problema metafísico alternativo: el universal accidental. La respuesta de Mumford para reemplazar a las leyes se encuentra en una metafísica de poderes y propensiones: ellos son capaces de ocupar el mismo rol que las leyes, pero siendo inmunes al Dilema Central y a las imputaciones de quidditismo, un Realismo sin Leyes (RSL), como él gusta dar en llamar a su propia posición.

El objetivo de este trabajo es mostrar que ni el Dilema Central ni el resto de los argumentos de Mumford contra el RN justifican su rechazo. En la 
sección 2 brindo un panorama general del debate entre realistas y antirrealistas nomológicos, revisando sumariamente algunas posiciones filosóficas que ejemplifican uno y otro bando, daré cuenta, concretamente, de los lineamientos generales del humeanismo como base de las alternativas que se enrolan en el AN (apartado 2.1) y de las propuestas realistas de Brian Ellis y David Armstrong (apartado 2.2). Ese recorrido prepara el escenario para exponer, en la sección 3, la primera batería de argumentos que Mumford dirige contra el RN. Analizo y discuto las imputaciones de quidditismo (3.1) y el problema del universal accidental (3.2). En la sección 4 doy cuenta de los aspectos fundamentales del Dilema Central y sus consecuencias más salientes respecto del rechazo del $\mathrm{RN}$, asimismo procuro discutir ese argumento a fin de mostrar que su eficacia depende de cierta nociones de 'gobierno' (o, más laxamente, 'rol determinante') y que el realista nomológico podría resistirse a aceptar.

Por último, la sección 5 incluye las conclusiones y comentarios finales a este trabajo.

\section{La cuestión metafísica de las Leyes Naturales}

La bien conocida distinción entre leyes científicas y generalizaciones accidentales monopolizó en buena medida las primeras discusiones en torno a la cuestión de las leyes. Se trataba entonces, a pesar de las múltiples inflexiones de la discusión, de respaldar conceptualmente la diferencia intuitiva que existe entre correlaciones necesarias entre propiedades (por ejemplo entre 'ser un metal' y 'dilatarse con el calor') y correlaciones factualmente constantes, pero contingentes (por ejemplo entre 'ser un jugador de los Chicago Bulls' y 'medir más de 1,50m.'). A pesar de ello, la elucidación de la naturaleza de esa necesidad era un tópico más bien secundario respecto de las consideraciones semánticas, sintácticas y lógicas que tenían por objeto brindar un conjunto de condiciones suficientes para que un enunciado pudiera ser considerado como perteneciente a la primera categoría. Contrariamente, la discusión entre realistas y antirrealistas nomológicos es hoy día, en sus bases, de orden metafísico. Hay muchas maneras de esbozar un mapa de ese debate, y ya que en las subsecciones siguientes daré cuenta de la posición de algunos de sus actores principales, me limitaré ahora a imagen puramente conceptual de la cuestión. 
Imaginemos tres pequeños mundos posibles, llamémoslos $\mathrm{M}_{0}, \mathrm{M}_{1}$ y $\mathrm{M}_{2}$. $\mathrm{M}_{0}$ está compuesto por un puñado de particulares y propiedades que los primeros instancian; digamos, particulares a, b y c, y propiedades $F$ y $G$, supongamos también que todos (particulares y propiedades) son observables. En ese mundo resulta siempre verdadero que ' $\mathrm{Fa}$ ', ' $\mathrm{Fb}$ ', ' $\mathrm{Ga}$ ' y ' $\mathrm{Gb}$ '. $\mathrm{M}_{1}$ y $\mathrm{M}_{2}$ son mundos posibles exactamente idénticos a $\mathrm{M}_{0}$ en todos los aspectos descriptos hasta ahora ${ }^{2}$, sin embargo mientras que en $\mathrm{M}_{0}$ todos los Fs son Gs de manera puramente contingente, en $\mathrm{M}_{1}$ lo son necesariamente. Podría pensarse que la diferencia radica realmente en que en $\mathrm{M}_{0}$ habrá, en algún momento, un particular que sea $F$ pero deje de ser $G$, mostrando así la contingencia de la regularidad; pero ese no es el caso en nuestro ejemplo, $\mathrm{M}_{0}$ es tal que en toda su historia resulta que todos los Fs son Gs, siempre y sin excepción. Creo que cualquiera puede obtener una interesante información acerca de su filiación filosófica preguntándose si encuentra que existe en verdad una diferencia entre $\mathrm{M}_{0}$ y $\mathrm{M}_{1}$, o se trata en realidad del mismo mundo. Con todo, y a pesar de contener conexiones necesarias, $\mathrm{M}_{1}$ no es todavía un mundo de leyes. $\mathrm{M}_{2}$, entonces, no sólo posee la misma configuración de relaciones necesarias que M1 (configuración que Mo posee sólo contingentemente), sino incluye además un rasgo extra: leyes naturales que soportan dicha necesidad. En qué consisten exactamente esas leyes es materia de controversia, para algunos (e.g. Armstrong) las leyes son universales de segundo orden que se instancian como relaciones entre universales de primer orden (en este caso, F y G), para otros (e.g. Ellis) se trata de propiedades esenciales de las clases naturales de ese mundo, es decir, de la adición de un rasgo de esencialidad a dichas propiedades. Debe notarse que $\mathrm{M}_{0}, \mathrm{M}_{1}$ y $\mathrm{M}_{2}$ son mundos empíricamente equivalentes, ningún observador interno o externo notaría alguna diferencia en ellos en tanto sistemas de entidades considerados sincrónicamente, o respecto de su desarrollo en el tiempo. Sin embargo, si se aceptan sus matices metafísicos, se trata de tres ontológicamente bien diferenciados.

Si bien la cuestión metafísica acerca de las leyes naturales ha tenido muy diversas aristas cuyo relevamiento resultaría imposible en este breve

\footnotetext{
2 La configuración de las ontologías de estos extraños mundos tiene como único propósito eliminar todo elemento que pueda desviar la atención del punto que pretendo establecer, sin embargo, el mismo argumento puede aplicarse a nuestro mundo real y los mundos posibles equivalentes correspondientes.
} 
cuadro, puede decirse que para el $\mathrm{AN}$ humeano nuestro mundo es semejante a $\mathrm{M}_{0}$, para el RSL semejante a $\mathrm{M}_{1}$, y para los realistas nomológicos, alguna versión de $\mathrm{M}_{2}$. Puesto en otros términos, el RSL impugna el cuadro metafísico que presentan $\mathrm{M}_{0}$ y $\mathrm{M}_{2}$, el primero por considerarlo carente de toda motivación filosófica, el segundo, apelando al mencionado Dilema Central. Previo a adentrarnos en este último punto, revisemos brevemente las posiciones contra las que Mumford dirige su argumentación, incluyendo algunos nombres propios.

\subsection{Humeanismo}

Para quienes defienden una metafísica humeana, la pintura metafísica de la realidad podría describirse del siguiente modo: el mundo está constituido por eventos discretos, cada uno de ellos se autocontiene y es tanto ontológica como causalmente independiente del resto. He aquí una analogía que puede resultar ilustrativa: el mundo es como un mosaico cuyas piezas son estos eventos discretos y causalmente inertes, donde cada pieza es lo que es por sí misma, independientemente del resto, muchas de ellas se presentan en relaciones regulares de semejanza y contigüidad espaciotemporal, pero fuera de ellas ninguna relación de necesidad las conecta. Eso es nuestro mundo, una cosita y luego otra (Lewis, 1986:ix). La analogía es pertinente en un aspecto más: la independencia de cada una de las piezas no implica que no haya regularidades; por el contrario, con la dedicación suficiente podemos encontrar diversos y complejos patrones en la distribución de las piezas, podemos descubrir que toda pieza roja es contigua de una hexagonal, o que a cada grupo de piezas semejantes en color le sigue un número de piezas verdes determinable por alguna función, etc. El punto es que ninguna de esas regularidades identificadas por la investigación empírica está determinada por una necesidad interna (de una pieza implicando a otra) ni externa (del sistema total implicando alguna distribución).

Las propuestas en esta línea suelen ser agrupadas bajo el nombre de 'teorías regularistas', pero a pesar de que en ocasiones el regularismo es presentado como una teoría de acerca de las leyes (Psillos 2002, p. 137), se trata en realidad de una teoría acerca de las regularidades, o las conjunciones constantes en la naturaleza. Del mismo modo propuestas como la de Cohen y Callender (2009) — según la cual las leyes son tan sólo los axiomas del mejor 
sistema descriptivo del mundo, llamada por tanto Teoría de los Mejores Sistemas - caen bajo antirrealismo respecto de las leyes. Dicho de otro modo, según las consideraciones expuestas sobre la cuestión metafísica de las leyes, tanto la pura teoría regularista de las leyes como la Teoría de los Mejores Sistemas se enmarcan, a pesar de lo que declaren sus propios defensores, dentro del AN.

Las reservas de Mumford para con el regularismo (principalmente dirigidas a su versión más acabada, defendida por Lewis) se fundan en una evaluación motivacional. Su aceptación descansa en la adopción de una cierta pintura metafísica, si esa pintura metafísica es aceptada, la teoría se sigue adecuadamente (Mumford 2004, p. 49). El problema del regularismo radica entonces en la metafísica humeana en la que se sustenta, pero, a su vez, la crítica hacia ella no puede ser de orden lógico o conceptual. La posición es consistente, e irrefutable por apelación a intuiciones tales como la distinción entre verdaderas leyes y generalizaciones accidentales, intuiciones que, por principio, el regularista no comparte. El punto de Mumford consiste en señalar que una ontología de unidades discretas y causalmente inertes, sin vínculo alguno de necesidad "no resulta una metafísica atractiva, y además, no podemos encontrar razones convincentes para aceptarla." (2004, p. 30)

El anterior no es, sin dudas, un argumento concluyente contra regularismo. Quizás ni siquiera sea un buen argumento. De hecho los humeanos pueden — como frecuentemente lo hacen-invocar a su favor el principio de economía que indica no multiplicar nuestra ontología sin necesidad. Pero no es ese el foco de este trabajo, por lo que será mejor continuar nuestra exploración de la polémica en torno a las leyes naturales.

\subsection{Esencias y universales}

Mumford cree que Brian Ellis cree que las leyes existen, pero son internas a sus instancias, más precisamente coinciden con las propiedades esenciales las clases naturales ${ }^{3}$. En palabras del propio Ellis:

\footnotetext{
${ }^{3}$ A pesar de lo dicho hasta aquí, Ellis ha negado que su filosofía lo comprometa con el RN. Reseñando el libro de Mumford, afirmó: "Efectivamente hablé en ocasiones de las leyes de la naturaleza como 'gobernando' varias clases de fenómenos. Aquella fue sólo una manera descuidada de hablar, de la que me disculpo". Y agrega: "Las leyes de la naturaleza no son cosas en el mundo, sino proposiciones que describen la clases de
} 
todas las leyes de la naturaleza, desde las más generales (...) hasta las más específicas (...) derivan de las propiedades esenciales de los objetos y eventos que la constituyen, y deben regir en cualquier mundo de la misma clase natural que el nuestro. (Ellis, 2011:4)

La cita tiene matices algo conflictivos, pero es leída como una afirmación de que, dado que el mundo tiene una estructura jerárquica de clases naturales, las relaciones de necesitación entre propiedades están dadas por su carácter esencial y constituyente de esas clases. Es así entonces que "hay una ley de la naturaleza donde quiera que haya una propiedad esencial de una clase natural" (Mumford, 2004: 108). Esta metafísica supone un "rechazo de la posición nominalista básica que permite que dividamos el mundo a través de nuestros conceptos derivados de la mente", según la cual "no puede haber ninguna afirmación de correspondencia entre nuestros esquemas conceptuales y la realidad no conceptual" (Mumford, 2004: 107). Asimismo, implica un rasgo importante respecto del contraste con otras formas de $\mathrm{RN}$ : dado que la estructura nómica del mundo se corresponde con su estructura de clases naturales, y que ésta se define a partir de propiedades que son esenciales a esas clases, las leyes de la naturaleza resultan necesarias en este esquema.

Puesto que las leyes coinciden con las propiedades esenciales de las clases, no resultan externas a sus instancias. Esto es lo que motiva a Mumford a etiquetar la teoría de Ellis como una concepción internalista, en la que "las leyes no son externas a las cosas que gobiernan, no existen independientemente de ellas (2004: 105).

Como queda de manifiesto, el cuadro metafísico de Ellis depende de la aceptación tanto de una estructura jerárquica de clases naturales, como de la existencia de propiedades esenciales que determinan dicha estructura. Ambos o alguno de esos compromisos podrían juzgarse inadecuados no infundados como veremos, esa será al menos parcialmente la estrategia de Mumford—, y si ese es el caso el defensor del RN deberá buscar una base para su posición en una metafísica diferente.

necesidades naturales que existen en él (...) no son ítems que deban estar presentes en el ontología de nadie”. (Ellis 2006, p. 438). No obstante ello podría pensarse que la concepción internalista acerca de las leyes es, a pesar de las declaraciones de Ellis, una posición disponible para un realista nomológico.

Manuscrito - Rev. Int. Fil., Campinas, v. 38, n.3, pp. 59-80, set.-dez. 2015. 
Otra alternativa es entonces adoptar la concepción externalista de las leyes defendida por Dretske (1977), Tooley (1977) y Armstrong (1983) (de aquí en más DTA). La DTA sostiene que las leyes son un tipo de universal de segundo orden, en esta geografía metafísica las propiedades son entendidas como universales efectivamente instanciados, que mantienen entre sí diversas relaciones de necesitación; esas relaciones de necesitación son las leyes. Si tener la propiedad $F$ implica tener la propiedad $G$, eso se debe a que $F$ y $G$ están vinculados en una relación $\mathrm{N}$ de necesidad tal que $\mathrm{N}(\mathrm{F}, \mathrm{G})$. Las leyes, por tanto, no son más que universales de segundo orden que instancian en universales de orden inmediatamente inferior. Pese a ello, no hay para Armstrong más que una relación posible entre universales de primer orden: la relación de necesitación nómica que ' $\mathrm{N}$ ' simboliza.

Toda esta metafísica adopta una forma del realismo moderado aristotélico, por lo que es inmanentista: no habría universales F ni $G$ si no hubiese entidades en el mundo en las que $F$ y $G$ se instancien, como no habría relación $\mathrm{N}$ de necesitación (es decir, ley) si no hubiesen instancias en las que efectivamente $F$ haga necesario a $G$. El rechazo de los universales no instanciados implica rechazar la idea de que a cada predicado general le corresponda un universal. Armstrong se siente cómodo con dicha consecuencia, toda vez que no pretende sostener que la identidad de los universales se determina semánticamente, sino un Realismo a posteriori acerca de universales. Para esta posición, determinar qué universales hay (es decir, qué propiedades y relaciones repetibles hay en el mundo) es algo que debe hacerse a posteriori sobre la base de la ciencia total. Por otra parte, este criterio de identidad para los universales excluye universales disyuntivos o negativos, así como la propiedad de ser una cosa idéntica a sí misma, relegándola a la categoría de pseudo-propiedad. Esto último, en razón de que la identidad no tiene ningún poder nómico o causal. Otra característica saliente, importante para algunas consideraciones que se expondrán en la próxima sección, es que la relación $\mathrm{N}$ resulta nomicamente necesaria, pero metafísicamente contingente. Es decir, mientras que centrada en un mundo relaciona de modo (nómicamente) necesario dos universales de orden inferior, pueden existir mundos posibles en los que esos mismos universales no estén vinculados por una relación de necesitación, por lo que $\mathrm{N}$ (aplicada a dichos universales) no resulta metafísicamente necesaria (Armstrong, 1983: cap. 11). Como hemos visto, la propuesta de Ellis se diferencia en este sentido de la DTA. 
Esta propuesta pretende brindar una explicación metafísica de aquello que el regularismo humeano trata como un mero factum. Tal como Mumford lo reconoce, "[] principal innovación de la DTA fue la afirmación de que las leyes eran acerca las relaciones entre universales o propiedades, y sólo indirectamente respecto de los particulares que instancian esas propiedades" (2004: 85).

\section{Mumford vs. el Realismo Nomológico, acto I: universal accidental y quidditismo}

Como se ha anticipado, la estrategia final de Mumford será derribar de un solo golpe el esencialismo de Ellis y la DTA, mostrando que ambas posiciones caen bajo el Dilema Central. Así y todo, se ocupa de brindar razones que pretenden socavar separadamente cada una de las propuestas. Es así que sostiene que la concepción internalista ha de enfrentarse a ciertas dificultades para justificar algunos de sus presupuestos metafísicos, principalmente, a lo que llama 'el problema el universal accidental'. El RN externalista, por su parte, acaba por implicar una tesis indeseable: el quidditismo acerca de las propiedades. Veamos ambos problemas en orden.

\subsection{Todos, y algo más que todos: el problema del universal accidental}

Como he procurado resaltar, el esencialismo de Ellis se asienta sobre dos columnas principales: la tesis que afirma la existencia de clases naturales, y la que afirma la existencia de propiedades esenciales que definen esas clases. Mumford tiene una actitud dispar respecto de ambas: "sostengo que el caso a favor de la existencia de las clases naturales es convincente, mientras que el caso a favor de las propiedades esenciales no lo es tanto" (2004: 106).

Sus reservas respecto de la segunda tesis son variadas. En primer término procura socavar distintos intentos de derivar plausiblemente la existencia de esencia a partir de la experiencia, la práctica científica o el fenómeno de la referencia. Pero aun cuando concluye que estas empresas conducen al fracaso, no descarta que "un camino más seguro a la esencia pueda 
encontrarse en algún momento futuro (...) por lo que una prueba aún no descubierta de la teoría no puede descartarse" (2004: 106).

No obstante ello, lo que sí resulta fatal para la tesis esencialista de Ellis es lo que denomina 'el problema del universal accidental'. Mumford (2004: 116) nos pide que supongamos la existencia de una clase natural definida por un conjunto de propiedades $\mathrm{K}$, de modo que todos los miembros de dicha clase poseen tales propiedades. Pero también podría suponerse que por obra y gracias del azar, por mera coincidencia (en el mismo modo que el regularista podría concebirlo) todos los miembros de dicha clase natural (pasados, presentes y futuros) poseen además un conjunto de propiedades Q. Claramente es posible que dicha posesión es accidental, pero todos los miembros $a, b, c \ldots$ z de $\mathrm{K}$ instancian Q. De ese modo, si ese fuera el caso

no tenemos forma de saber que entre las propiedades $P_{1}, P_{2}, P_{3}, \ldots P_{n}$, de todos los miembros de $\mathrm{K}$, no se esconde una propiedad universal, pero así y todo accidental. Dada la contingencia de las propiedades accidentales, nada parece descartar la posible universalidad de lo accidental. Esta posibilidad podría ser muy grave para el esencialismo. Ella sugiere que debe haber más en ser una propiedad esencial que simplemente ser una propiedad que posee cada miembro de clase. (Mumford, 2004: 116-7)

De este modo, a criterio de Mumford, la distinción entre propiedades esenciales y accidentales quedaría disuelta. Reconstruirla, según entiende, requiere que las propiedades constitutivas de clase sean instanciadas por todos sus miembros posibles de la clase. Pero si bien esa solución puede ser aceptable para alguna forma de esencialismo, no lo es — señala Mumford- para la defendida por Ellis, que tiene a las esencias y la necesidad por rasgos inmanentes de nuestro mundo.

Pero ese no es de hecho el único camino para sortear este problema, ni siquiera el más natural. Sostengo que el universal accidental no es en realidad un problema para el esencialismo, ya que se encuentra viciado desde su mismo planteo. Para mostrar dicho punto es preciso comenzar con una discriminación necesaria. Por una parte tenemos a los argumentos a favor de la existencia de propiedades esenciales. Mumford rechaza alguno de ellos, es indiferente a otros, y admite la posibilidad de que nuevas vías de razonamiento sean exploradas, pero lo que debe destacarse es que el objeto de esos argumentos es 
sustentar una conclusión metafísica. Ahora bien, una vez que el esencialista ha asumido ese compromiso con tales o cuales argumentos, la cuestión de la existencia de las esencias resulta independiente de grado y tipo de conocimiento que tengamos de ellas. Dicho de otro modo, es preciso distinguir respecto del carácter esencial de una propiedad la dimensión metafísica de la epistemológica. Mumford, por el contrario, confunde ambas en el planteo mismo de su objeción, y es por ello que su argumento falla en instituirse como un auténtico problema para el esencialismo. Las propiedades $\mathrm{K}$ son aquellas sin las cuales una entidad no podría ser un auténtico miembro de $\mathrm{K}$, no simplemente aquellas que son comunes a todos los miembros. La existencia de propiedades esenciales es independiente de si en efecto existe o no un test empírico para determinar si una propiedad es o no esencial. Por lo tanto, el hecho de que no tenemos forma de saber si una propiedad universal es esencial no alcanza para socavar la propuesta esencialista.

Podría pensarse que la posibilidad del universal accidental, incluso no siendo un problema fundamental para el esencialismo en tanto doctrina metafísica, le resta cierta plausibilidad al dejar empíricamente subdeterminado el acceso epistémico a las auténticas esencias. Esa dificultad no es sin embargo ajena a casi cualquier doctrina metafísica. El propio disposicionalismo de Mumford, de hecho, la replica en el caso de las disposiciones no efectuadas. Si bien no es el objeto de este trabajo ahondar en dicha alternativa, basta con señalar que para el disposicionalismo es posible que un poder o disposición real de un objeto permanezca eternamente como no-manifestado. Es por ello que es posible en ese marco - tanto como el universal accidental es posible en el marco del esencialismo- que los poderes reales de una entidad resulten empíricamente inaccesibles para nosotros.

\subsection{Lo que hacen, y algo más que lo que hacen: quidditismo.}

Según Armstrong, los universales de segundo orden que constituyen las leyes naturales son inmanentes, es decir, abstracciones a partir de particulares que los instancian. De ese modo no habría universales sin particulares que los instancien, como no habría leyes sin al menos un caso positivo. Es precisamente en este punto en el que Mumford encuentra la primera debilidad de la teoría DTA: esa inmanencia es una forma (en el mejor de los casos limitada) de superveniencia. La existencia real de una ley depende de (deriva, 
está determinada por) la instanciación efectiva de una secuencia causal entre dos universales cuya existencia real depende de (deriva, está determinada por) la instanciación efectiva de esas propiedades en el mundo. Por tanto, cabe preguntar si $\mathrm{N}$ no es después de todo es una relación sui genreis, casi un deus ex machina que pone sobre las propiedades la necesidad que ellas mismas no pueden brindarse. En algún sentido, no distaría mucho de ser una hipótesis ad hoc que permite justificar la estructura nómica del mundo (Mumford, 2004: 93).

Pero hay para la DTA, sin embargo, un problema aún más acuciante que deriva de su postulación de un carácter metafísicamente contingente para las leyes. Para Armstrong, las leyes son necesarias en un sentido nomológico, es decir, son ellas mismas una conexión necesaria entre dos universales que ocurre en el mundo real. Sin embargo esa relación no tiene por qué replicarse en todo mundo posible, por lo que hay en algunos de ellos relaciones de necesitación entre universales que son diferentes a las que existen en el mundo actual. De ello se desprende que el hecho de que algo sea una ley no es metafísicamente necesario, es decir, una distinción tajante entre necesidad nomológica y necesidad metafísica. ¿Qué hay de malo con esa concepción? El problema es que asume implícitamente una metafísica de propiedades categóricas. Una propiedad F que en el mundo actual causa necesariamente a la propiedad $G$ podría no hacerlo en, pongamos por caso, $\mathrm{M}_{3}$, y causar, en cambio, $Z$. Esa propiedad podría de hecho no hacer en $\mathrm{M}_{3}$ nada de lo que hace en este mundo, y ser aún la misma propiedad F; es decir, la identidad de una propiedad es independiente de su rol o perfil causal. Dependerá entonces de algo más; pero eso será todo lo que podamos decir: lo que hace a $\mathrm{F}$ ser la propiedad que es, es un "algo", una quidditas que subyace a su rol causal. Esa es precisamente la tesis del quidditismo: existe una naturaleza intrínseca, empíricamente inaccesible e independiente de su perfil causal que hace que una propiedad sea la propiedad que es.

La identidad de una propiedad debe ser independiente de cualquier cosa que resulte dependiente de las leyes. Lo único que parece escapar a esta red de leyes, y que podría determinar la identidad de una propiedad a través de cambios en las leyes, es una esencia individual de cada propiedad, una quidditas. (Mumford, 2004: 151)

Así, la tercera razón que hace indeseable a la concepción externalista de las leyes es que implica un quidditismo respecto de las propiedades. 
Sostengo, sin embargo, que varios puntos comprometen este diagnóstico. En primer lugar no es obvio que toda forma de la teoría DTA deba implicar el quidditismo. Como Mumford observa, esta tesis se desprende de la afirmación de Armstrong acerca del carácter contingente de las leyes naturales a lo largo de los diferentes mundos posibles. Pero ese compromiso dista de ser central para la concepción externalista de las leyes. De hecho este carácter contingente de las leyes no es suscripto explícitamente ni por Dretske ni por Tooley. Y aun si así hubiese sido, sería posible suprimir dicha tesis de la formulación de la DTA sin alterar sustancialmente su visión de las leyes como universales.

En segundo término, si bien Mumford insiste en calificar negativamente al quidditismo como un problema, una "consecuencia altamente indeseable" (2004: 146), un "peligro" (2004: 197) o una "tesis increíble" (2004: 158), hace muy poco por explicitar los motivos por los que dicho compromiso debería evitarse. El quidditismo implica que la naturaleza de muchas propiedades ha de resultar incognoscible, lo que Lewis llamó 'Humildad Ramsiana' (Ramseyan Humility). Lewis no tiene inconvenientes con la tesis de la Humildad, por lo que acepta sin problemas las quidditas. Del mismo modo Psillos afirma que está lejos de ser obvio que el quidditismo sea una tesis incorrecta, o al menos preferible a sus alternativas (Psillos, 2012: 174). El único argumento positivo de Mumford respecto de la inconveniencia del quidditismo parece ser el siguiente.

Podría haber dos mundos que coincidan en sus historias, pero difieran en sus propiedades fundamentales — diferentes debido a sus quidditas individuales- jugando los mismos roles nómicos. Pero, entonces, para cualquier número de propiedades (fundamentales) que se crea que puede haber, el quidditismo no tiene en principio ninguna razón para afirmar que no podría haber una más de lo que indica ese número. Más propiedades siempre pueden ser generadas simplemente mediante la sustitución de una propiedad por otra que juega el mismo papel, pero difiere en sus quidditas. (Mumford, 2004: 152)

Pero nuevamente Mumford mixtura inadecuadamente cuestiones metafísicas con cuestiones epistemológicas. Que dos mundos puedan ser empíricamente equivalentes pero diferir en el conjunto de sus propiedades fundamentales no implica que nosotros seamos capaces de generar propiedades 
fundamentales a partir de nuestro conocimiento o nuestra imaginación. Eso no es quidditismo sino idealismo, en el mejor de los casos. Lo que dicha posibilidad implica es que no podríamos conocer las naturalezas intrínsecas por las que dichos mundos difieren. Es decir, implica una forma de la tesis de la Humlidad defendida por Lewis. Mumford nos debe un argumento para mostrar que dicha tesis es increíble, un peligro o una consecuencia indeseable para la DTA.

Por último, incluso asumiendo que el quidditismo debería ser evitado, no es en absoluto claro que la alternativa propuesta por Mumford al RN -el RSL—pueda hacerlo. Las propiedades, según el RSL, no son más que (es decir, están constituidas por) un cluster de poderes y propensiones. Así la identidad de una propiedad no es independiente de su perfil causal, sino a la inversa: si se modificase éste último ya no estaríamos frente a la misma propiedad. La identidad de una propiedad se determina por extensión, por los poderes que la constituyen. Poderes reales, independientes de su manifestación efectiva. Pero cabe preguntar entonces: ¿en dónde reside la identidad de un poder? La identidad de un poder no puede estar en la propiedad que ese poder constituye, si así fuese se estaría cayendo en literalmente el mismo tipo de quidditismo que se denuncia en el RN. ¿Podría, en cambio, cada poder encerrar en sí mismo el principio de su propia identidad? Un poder hace o haría ciertas cosas en ciertas circunstancias. Tiene, digamos, ciertas Implicaciones Fácticas (IF), manifestadas o no. La identidad de un poder, si no podía residir en la propiedad de la que es parte, o bien reside en el conjunto de sus IF, o bien en alguna otra cosa. Lo segundo es inadmisible: si la identidad del poder se determinara por alguna otra cosa que el conjunto de sus IF, podría haber dos poderes con las mismas IF, es decir, dos poderes capaces hacer exactamente lo mismo, pero que aun así fueran dos. Y consecuentemente, podría haber dos propiedades distintas con exactamente el mismo perfil causal, lo que resulta contradictorio no sólo con lo dicho anteriormente, sino con el propósito elemental de esta propuesta. La naturaleza de un poder no puede entonces estar determinada sino por sus IF: lo que un poder es está determinado por lo que puede hacer. Con todo, no puede ser solamente eso. Si la naturaleza de un poder se agotase en sus IF, si no fuera nada más que ellas, volveríamos a la mera noción de "potencia" y no tendría sentido afirmar que un poder tiene algún tipo de realidad actual cuando no está manifestado. Tampoco habría modo de explicar cómo dos poderes que no están manifestados son dos de hecho poderes distintos. En nada diferiría el ser 
rompible de ser inflamable bajo el supuesto de que ninguno de ellos se actualizase jamás. El poder tiene que ser algo más que sus manifestaciones, algo numéricamente distinto aunque ontológicamente dependiente de ellas, un poder tiene que ser algo más que simplemente lo que puede hacer, algo que reúna al conjunto de sus IF como parte del mismo poder, distinto de otro. Literalmente un "algo", una esencia, una quidditas. Así, esta metafísica de poderes y propensiones tiene precisamente el problema que pretendía solucionar, y si el quidditismo del RN era una consecuencia indeseable, el de su propia metafísica es al menos tan indeseable como aquel.

\section{Mumford vs. el Realismo Nomológico, acto II: el Dilema Central}

Los realistas nomológicos eligen poner el peso de la necesidad en las leyes de la naturaleza, asumiendo que las regularidades observables tienen su fundamento en ellas, y que por lo tanto esas leyes son parte del elenco metafísico del mundo. Más allá de sus críticas puntuales a las distintas formas que este compromiso ha asumido que fueron objeto de la sección precedente, Mumford rechaza el RN apoyándose en su argumento estrella, el Dilema Central. Se trata de un argumento en dos pasos (Mumford, 2004: 144-5). En primer lugar se afirma que

(I) $\mathrm{O}$ bien las leyes naturales son [A] externas a los eventos que gobiernan, o bien son $[\mathrm{B}]$ internas a los eventos sobre los que juegan dicho rol gobernante.

Donde $[\mathrm{A}]$ y $[\mathrm{B}]$ representan respectivamente la DTA y la teoría esencialista de Ellis. El segundo paso consiste en mostrar la verdadera encrucijada que pone en jaque al realista nomológico.

(II) Si [A], entonces debe brindarse un relato plausible acerca de cómo tal rol de gobierno externo puede tener lugar; y si [B] debe hacerse lo propio respecto de un rol interno de gobierno de las leyes sobre sus instancias. 
Lo que resta es probar que dicho dilema es en efecto fatal para el RN. Debe notarse que para al menos empezar a considerar la pertinencia del argumento dos supuestos básicos deber ser aceptados:

(a) Las leyes han de tener una cierta adición de ser respecto de sus instancias.

(b) Las leyes deben gobernar sus instancias.

El primero implica que cualquiera que decida llamarse realista respecto de las leyes, debe comprometerse con que ellas tienen un espesor ontológico considerable, con que son cosas en el mundo, una categoría ontológica per se. El segundo supuesto implica que el rol de gobierno sobre sus instancias es necesario como un rasgo tanto constitutivo de las leyes en sí mismas, como de la viabilidad de una metafísica que las postule. Esto último significa que ambos supuestos funcionan además en el plano normativo como criterio de aceptabilidad para cualquier teoría acerca de las leyes naturales. Mumford se ocupa de aclarar que "puede tratarse de un sentido extremadamente débil de gobierno" y si la expresión nos resultara teóricamente incómoda, podríamos reemplazarla por cualquier nombre que denote "un rol (...) tal que el mundo hubiese sido diferente si [las leyes] no estuviesen en él" (2004: 145). Con todo, las leyes no pueden jugar ese rol, sea que se las considere externas o internas a sus instancias: si queremos escapar de Hume con la modalidad objetiva a cuestas, la puerta del RN no resulta una buena opción. La propuesta, entonces, será una metafísica de propiedades reductibles a clusters de poderes y/o disposiciones, capaces, según Mumford, de dar cuenta de sus relaciones de necesitación sin caer en las redes del Dilema Central.

En el caso de [A], la concepción externalista no es capaz de dar cuenta del rol gobernante de las leyes ya que éstas acaban por supervenir a sus instancias "en el sentido de que un universal es no nada por fuera de sus instancias y depende de sus instancias" y por ello no las gobiernan dado que "es muy poco plausible la suposición de que las leyes puedan gobernar algo sobre lo cual son supervenientes" (2004: 103). Vista con cuidado, es una objeción curiosa: el problema de la concepción externalista de las leyes es que sus leyes no son propiamente externas, por lo que no es propiamente una concepción 
externalista de las leyes. Además, nuevamente, el argumento de Mumford toma por un rasgo general de la DTA algo que (en el mejor de los casos) es únicamente aplicable a posición de Armstrong, a saber, que los universales no son nada por fuera de sus instancias. Eso es radicalmente falso en el caso de Tooley (quien abraza un platonismo acerca de los universales) implausible en el caso de Dretske (quien contempla la posibilidad de leyes no instanciadas) e incluso discutible en el de Armstrong, ya que su realismo aristotélico difícilmente implique que la identidad de un universal se agote en sus instancias, y que por tanto el primero tenga un carácter metafísicamente inerte respecto de las segundas.

En cuanto a [B], en el marco del internalismo las leyes son caracterizadas a partir de las propiedades esenciales constitutivas de las clases naturales. Como he procurado mostrar, los ataques de Mumford contra la existencia de tales propiedades resultan insuficientes para socavar esta posición. Sin embargo el Dilema Central es presentado como un obstáculo infranqueable para el esencialismo.

Dos opciones surgen para terminar de elucidar el real compromiso metafísico del esencialismo: o bien las leyes son reductibles a las propiedades esenciales constitutivas de clase, o bien son algún tipo de entidad que superviene a la estructura de clase que esas propiedades definen. Pero, según Mumford, tanto "si las leyes supervienen a cosas que no son leyes, o si están constituidas por ellas (...) necesitamos un relato acerca de cómo pueden gobernar o desempeñar algún rol desde dentro", y el problema es que "en tal relato parece altamente implausible que dichas leyes gobernantes sean internas a las cosas que gobiernan" (2004: 153-4). Nuevamente, señalar la impasibilidad de que una entidad superveniente gobierne aquello sobre lo cual superviene es presentado por Mumford como una prueba de que el Dilema Central es fatal para el realista nomológico.

Pero según entiendo, ese no es realmente el caso. Ordenemos un poco la argumentación: las leyes pueden entenderse como internas a sus instancias en dos sentidos, o bien como constituidas por ellas, o bien como supervenientes a ellas. En cualquiera de los dos casos esta caracterización de las leyes incumple el segundo requisito de aceptabilidad que se había impuesto al RN: las leyes no gobiernan a sus instancias dado que es muy poco plausible la suposición de que las leyes puedan gobernar algo sobre lo cual son supervenientes o a lo cual son reducibles. El problema aquí es: ¿qué es exactamente lo que gobierno quiere decir en este 
contexto? O, en una versión más relajada, ¿qué es lo que con rol determinante se quiere significar? No creo poder contestar esas preguntas del modo correcto, a decir verdad, tampoco creo que Mumford pueda hacerlo. Pero permítanme contar algunas historias. Tomemos una estufa eléctrica, a decir verdad, un aparato muy sencillo. El termostato de la estufa funciona a partir de algunas piezas de metal con diferente coeficiente de dilatación, de modo que la propia temperatura de la estufa modificaba esas piezas accionando así un dispositivo de apagado o encendido. El termostato no es idéntico a la estufa, aunque ciertamente es parte de la estufa; la cual no funciona sin él, que, por otra parte, tampoco funciona sin la estufa. El caso es que en algún sentido interesante de 'gobernar' tendemos a decir que el termostato gobierna la temperatura de la estufa, aun cuando funcionalmente dependa por completo de ella. Sin embargo, más allá de que es claro que ello califica como un rol determinante, puede pensarse que la analogía es débil, ya que el modo en que las leyes dependen ontológicamente de sus instancias no es el mismo en el que un termostato depende del artefacto en el que está instalado. Así que contaré otras historias. Muchos filósofos se ganan la vida defendiendo la idea de que los procesos mentales son epifenómenos de los cerebrales, de que supervienen a ellos; aun así, muchos de esos filósofos sostendrían que en algún sentido interesante los fenómenos mentales gobiernan, o cumplen algún rol determinante respecto de ciertos fenómenos cerebrales. Las reglas o normas que rigen sobre el uso de nuestro lenguaje dependen de —están determinadas por, derivan de, o tal vez incluso están constituidas por- nuestras prácticas lingüísticas, con todo esas prácticas están gobernadas por dichas reglas, y éstas juegan un rol determinante en nuestros modos de comunicarnos. Algo semejante podría decirse de las leyes, en el sentido jurídico del término.

No pretendo aquí afirmar sustantivamente nada acerca de cómo funcione el lenguaje, o la mente humana, o una estufa eléctrica, sino simplemente señalar que si se quiere objetar la posibilidad de que entidades supervenientes tengan cierto rol relevante respecto de aquellas entidades sobre las cuales supervienen, debe hacerse mucho más que un simple diagnóstico de falta de plausibilidad. Ese no resulta un argumento aceptable, y a decir verdad ni siquiera es un argumento.

Puesto en términos más precisos: sea $\mathrm{E}$ un conjunto de entidades cualesquiera $e_{1}, e_{2}, \ldots, e_{\mathrm{n}}$, del tipo que uno aceptaría de modo no problemático en su ontología. Sea $\mathrm{S}$ una entidad que o bien superviene a $\mathrm{E}$ o bien simplemente 
está constituida por E. En ambos casos, es claro, S tiene una relación de dependencia ontológica respecto de E, es decir, $\mathrm{S}$ no podría existir si no existiese E. Menos obvio, aunque todavía razonable, resulta pensar que $S$ tenga lo que podría llamarse una relación de dependencia causal respecto de $\mathrm{E}$, es decir, que todo cambio en E conlleve un cambio en S. Eso es indudable en el caso de la mera constitución, pero - aun cuando muchos definen la superveniencia de ese modo- no es claro más allá de toda duda que dicha relación se cumpla en el caso de la superveniencia. Podría pensarse por ejemplo que si el lenguaje como entidad superviene a las prácticas individuales, no todo cambio en las prácticas conlleva un cambio en el lenguaje. Pero pese a ejemplos de este tipo demos por hecho que la dependencia causal también se cumple. Lo que no resulta en modo alguno obvio, y ni siquiera razonable hasta donde puedo ver, es la afirmación de que $\mathrm{S}$ no puede jugar ningún tipo de rol relevante respecto de $\mathrm{E}$. $\mathrm{El}$ hecho de que $\mathrm{S}$ dependa ontológica e incluso causalmente de $\mathrm{E}$ no implica ni lógica ni conceptualmente que $\mathrm{S}$ no pueda afectarlo de modo alguno. Los ejemplos ofrecidos más arriba, a pesar de no ser concluyentes, sugieren de modos diversos ese punto. $\mathrm{E}$ incluso si fueses cuestionables, es claro que Mumford nos debe un argumento para mostrar que $\mathrm{S}$ es incapaz de jugar un rol relevante respecto de $\mathrm{E}$, uno más fuerte que un mero diagnóstico de implausibilidad.

\section{Conclusión}

Las leyes naturales son y seguirán siendo un tópico ineludible para toda reflexión filosófica acerca de la ciencia dada su vinculación con cuestiones de vital importancia como la modalidad, la causalidad y el Realismo Científico. El análisis metafísico muestra, sin embargo, que buena parte de las referencias a leyes corresponden a un discurso que omite algunas distinciones básicas: creer que la práctica científica tiene como una de sus características esenciales el formular leyes, así como creer que existe algún correlato objetivo para nuestro enunciados modales, no resultan condiciones suficientes para ser considerado un realista nomológico. El mismo Ellis, como hemos visto, ha admitido que el $\mathrm{RN}$ internalista que Mumford vio en su obra no era más que un modo poco cuidadoso de hablar. El combate contra el RN es, principalmente, uno contra la DTA. Tal como se ha concluido en las secciones 3 y 4, las razones de Mumford 
en su contra son insuficientes, en tanto dependen de una noción de 'rol gobernante' y supuestos acerca de la superveniencia que el realista nomológico podría — de hecho, debería - cuestionar. Además, tanto el problema del quidditismo como el del universal accidental no resultan obstáculos insuperables para el realista nomológico. $\mathrm{Y}$ aun si ese fuera el caso, el propio RSL aparece amenazado por dificultades semejantes.

De ese modo Mumford debe hacer más tanto para mostrar que el RN debe ser rechazado, como para hacer patente que su propia posición resulta verdaderamente superadora.

\section{Referencias}

Armstrong, D. M. What is a Law of Nature? Cambridge, Cambridge : University Press, 1983.

Chisholm, R. “The Contrary-to-Fact Conditional”. Mind No. 55, pp. 289307, 1946.

COHEN, J. and CALlender, C. "A better best system account of lawhood". Philosophical Studies, vol. 145, no 1, p. 1-34, 2009.

DreTSKe, F. "Laws of nature". Philosophy of Science No. 44, pp. 248-68, 1977.

ELLIS, B. Scientific Essentialism, Cambridge: Cambridge University Press, 2001.

- The Philosophy of Nature: A Guide to the New Essentialism, Chesha: Acumen, 2002.

- "Loking for laws - review symposium". Metascience, No. 15, pp. 437-469, 2006.

Goodman, N. "The Problem of Counterfactual Conditionals". Journal of Philosophy, No. 44, pp. 113-128, 1947.

Hempel, C. y Oppenheim, P. "Studies in the Logic of Explanation". Philosophy of Science, No. 15, pp. 135-175, 1948. 
LEWIS, D. Philosophical Papers II, Oxford: Oxford University Press, 1986.

MumFORD, S. Laws in Nature, London : Routledge, 2004.

PsiLlos, S. Causation and Explanation, Chesham: Acumen, 2002.

-. "Adding Modality to Ontic Structuralism: An Exploration and Critique", en E. Landry and D. Rickles (eds), Structure, Object, and Causality. The Western Ontario Seriesin Philosophy of Science. Dordrecht: Springer, pp. 169-85, 2012.

ToOLEy, M. "The nature of laws". Canadian Journal of Philosophy, No. 74. pp. 667-98, 1977. 